\title{
ANÁLISIS DE DIFERENTES ESTRATEGIAS DE CONTROL AUTOMÁTICO APLICADAS A UN PROTOTIPO DIDÁCTICO PARA EL ESTUDIO DE LA VENTILACIÓN DE MINAS SUBTERRÁNEAS
}

(Analysis of different strategies of automatic control applied

to a didactic prototype for the study of underground mines ventilation)

\section{Juan Mauricio Salamanca*, John Jairo Niño Merchán, ** Adrián Felipe Pérez *** \\ *Escuela de Ingeniería Electrónica, UPTC. Grupo DSP-UPTC. juan.salamanca@uptc.edu.co \\ ** Escuela de Ingeniería de Minas, Grupo DSP-UPTC. john.ninomerchan@uptc.edu.co}

(Recibido: 5 de Agosto de 2014 y aceptado 1 de Octubre de 2014)

\begin{abstract}
Resumen:
El presente artículo describe un prototipo desarrollado para el estudio y control de la ventilación para minería. Se presenta el modelado por medio de una identificación paramétrica y el resultado de la aplicación de estrategias de control básicas como el PID, On-Off y avanzadas como control basados en modos deslizantes.
\end{abstract}

Palabras clave: ventilación de minas, estrategias de deslizantes. control, control PID, control ON-OFF, control con modos

\section{Abstract:}

This paper describes a prototype developed for the study and control of ventilation for mining. Modeling is presented by means of a parametric identification and the result of the application of basic control strategies such as PID, On-Off and advanced as a control based on sliding modes.

Keywords: mine ventilation, On-Off control, PID control, sliding mode control.

\section{INTRODUCCIÓN}

Una de las mayores preocupaciones para los ingenieros de minas que laboran en minas subterráneas es mantener dentro de la operación un flujo de aire adecuado para ventilar la mina y así desarrollar de manera segura la explotación (Keith \& Wallace, s.f.; Reed \& Taylor, 2007; Salamanca, Niño \& Higuera, 2012). La manera más efectiva de ventilar una mina es mediante la instalación de ventiladores principales ubicados estratégicamente en la mina para generar un circuito de ventilación constante, el cual no será dependiente de la presión barométrica y la temperatura del aire en superficie, como sucede con la ventilación natural, la cual depende de estas variables ver (Reed \& Taylor, 2007).

La ventilación mecanizada actualmente tiene grandes retos para la ingeniería y uno de ellos es conseguir que se pueda continuar llevando aire de buena calidad a todos los rincones de la mina sin importar su profundidad, reduciendo los consumos de energía eléctrica y sin poner en riesgo la seguridad de la operación (Salamanca, Niño \& Higuera, 2012; Keith \& Wallace, s.f.; Witrant, Niculescu \& Marchand, 2008). Una parte de la solución del anterior problema está en el empleo de estrategias de control automático, las cuales pueden comandar los ventiladores de acuerdo con diferentes factores como cambios de temperatura y aumento de niveles de gas. Las anteriores variables pueden ser detectadas mediante sistemas de sensores que pueden enviar la señal a un sistema de comando y allí dar una orden al ventilador. Para más información ver (Witrant et al., 2008; Witrant, Niculescu \& Marchand, 2008; Olaru, Sandou, Witrant \& Niculescu, 2008; Di Benedetto, D'Innocenzo, Serra \& Witrant, 2008).
Dentro de las estrategias de control que se pueden utilizar para controlar los ventiladores en una mina, están:

Control PID.

Control On-Off.

Control por modos deslizantes.

Estas estrategias ofrecen flexibilidad en la implementación con el equipo existente, como variadores de velocidad PLC y procesadores digitales. Además, las estrategias ofrecen robustez frente a variaciones de parámetros y ruidos externos (Edwards \& Spurgeon, 1998) (Salamanca, Niño Merchán \& Higuera, 2012). En este artículo se presentan tres estrategias de control aplicadas en una mina prototipo. En ella se evalúa cuál es la más efectiva para evacuar los gases. El artículo está organizado de la siguiente forma. En la sección 2 se realiza la descripción del prototipo, en la sección 3 se muestra el modelado experimental de la planta; en la sección 4 se presenta el diseño e implementación del control PID; en la sección 5 se presenta el diseño e implementación del controlador ON-OFF, en la sección 6 se presenta el diseño y la implementación de la estrategia de control basada en modos deslizantes. Finalmente, en la sección 7 se relacionan las conclusiones.

\section{DESCRIPCIÓN DEL PROTOTIPO}

El diseño del prototipo se basó en el artículo Air Flow Modeling in Deep Wells: Application to Mining Ventilation de Witrant y Johansson (2008).

El tamaño del prototipo es de $1.0 \mathrm{~m}$. de altura por $0.70 \mathrm{~m}$. de ancho. Esta escala corresponde 1:100 y pertenece a una mina subterránea promedio, en la que el material de explotación puede ser carbón, hierro o caliza.
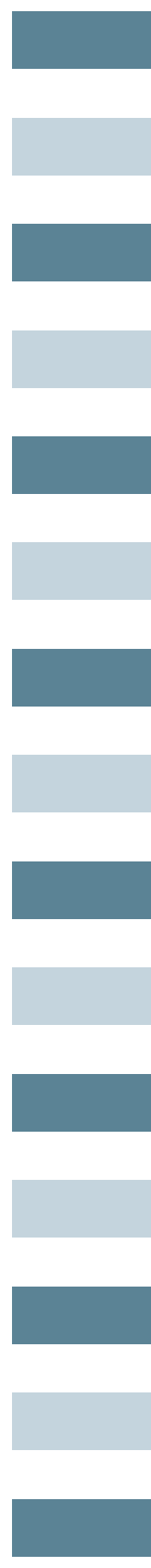
La mina prototipo tiene dos túneles de acceso que la comunican con la superficie. En la entrada de cada túnel hay un ventilador. En el túnel 1 hay un ventilador soplante, el cual ingresa aire a la mina; en el túnel 2 hay un ventilador aspirante que succiona el aire viciado para evacuarlo de la mina. Los dos túneles se comunican entre sí por dos niveles. El primer nivel cuenta con dos galerías A y B, en las cuales se simula una labor de explotación, labores que en una mina real son difíciles de ventilar. En esta galería se ubican sensores de $\mathrm{CO}$ y $\mathrm{CO} 2$ para la detección del nivel de gas. El aire que ingresa a estas galerías es llevado por dos ventiladores que toman el aire del circuito principal y lo inyectan a los frentes de explotación por medio de ductos de ventilación. El segundo nivel también comprende el mismo diseño. En este prototipo se asume que el sostenimiento minero es natural (el sostenimiento minero permite tener una excavación subterránea sin que se derrumbe), puesto que se desea tener un flujo de aire con pocas resistencias para analizar su efecto en los gases que se quieren diluir y la forma como estos son evacuados hasta el exterior de la mina. El control se implementó con la tarjeta de la National Instruments, y los sensores empleados fueron MQ-7, los cuales son muy sensibles al gas $\mathrm{CO}$ y CO2. En la Figura 1 se observa una imagen de la mina prototipo.

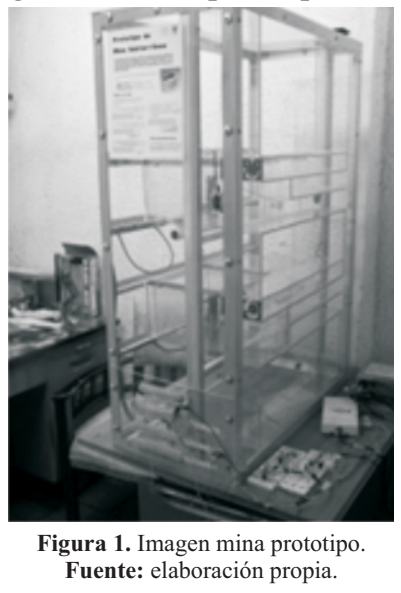

\section{MODELAMIENTO DE LA MINA PROTOTIPO}

En las ecuaciones para conocer el modelo del flujo de aire en la red de ventilación se emplearon las leyes de Kirchhoff (Yunan , Koroleva \& Miroslav, 2003; Viorel, 2011; Salamanca, Niño Merchán \& Perez, 2014).

Para obtener las curvas de reacción del sistema se realizó el siguiente procedimiento.

- Se hace una limpieza del circuito de ventilación con los ventiladores auxiliares y principales al máximo. Con ello se calibra el sensor de gases a su valor mínimo.

- Se apagan los ventiladores auxiliares.

- Se inyecta gas a los frentes de explotación hasta saturarlos, para calibrar los sensores a su valor máximo.

- Se pone en funcionamiento cada ventilador auxiliar por separado al $50 \%$ de su valor máximo y se espera que la salida de cada sensor se estabilice en un valor. En estas condiciones se realizan las mediciones de la constante de tiempo, la ganancia y el retardo de tiempo.

- $\quad$ Se aumenta la velocidad de cada ventilador por separado hasta el $100 \%$ de su valor máximo. Se espera que las salidas de los sensores se estabilicen y en estas condiciones se realizan las mediciones.

- Se realiza el procedimiento anterior, pero en este caso se parte con los ventiladores a su valor máximo.

De las pruebas realizadas se obtuvieron las siguientes funciones de transferencia:

\subsection{Motor uno (M1)}
$Y(S)=\frac{-0.5615}{104.025 S+1}$
$U(S)=\frac{-0.5615}{104.025 S+1}$

Versión muestreada suponiendo la entrada generada por medio de $\mathrm{ZOH}$ con $\mathrm{Tm}=1 \mathrm{seg}$.

$\begin{aligned} & Y(Z, T m) \\ & U(Z, T m)\end{aligned}=\frac{-0,005372}{Z-0,9904}$

3.2 Motor dos (M2)

$\begin{aligned} & Y(S) \\ & U(S)\end{aligned}=\frac{-0.589}{68.85 S+1}$

Muestreando las funciones de transferencia descritas por las ecuaciones (1), (2) y (3), suponiendo la generada por medio de $\mathrm{ZOH}$ con $\mathrm{Tm}=1 \mathrm{seg}$, obtenemos la función de de pulsos indicada en la ecuación (4)

$$
Y(Z, T m)=\frac{-0.008493}{Z-0.9856}
$$

Condiciones iniciales:

1. Ventilador principal soplante $: 3.7 \mathrm{~V}$

2. Ventilador principal extractor: $0 \mathrm{~V}$

3. Ventilador galería uno (M1):0,297 V

4. Ventilador galería dos (M2) : 0,502 V

$(0,297-3.3 \mathrm{~V})$

$(0,502-3.3 \mathrm{~V})$

PWM: [0-3,3] Voltios

Potencia: $2 P I R=20$

$R=\Omega$

\section{DISEÑO DEL CONTROLADOR PID}

Tal vez el controlador más conocido tanto en el ámbito académico como en el industrial es el PID, debido a su estructura flexible y a su fácil implementación. La ecuación general del PID es la siguiente (ecuación (5)):

$$
u(t)=K p\left[e(t)+\frac{L}{r_{i}} \int^{t}-\infty e_{(t)} d_{t}+r_{d} \frac{d}{d t} e(t)\right]
$$

Para el diseño solo se requiere un controlador PI $\left(T_{D}=0\right)$. La función $e(t)$ es el error entre la salida deseada o punto de ajuste y la salida actual del proceso.

Según lo anterior, la función de transferencia del controlador queda expresada por la ecuación (6):

$$
?_{0}=0,22 \frac{\mathrm{rad}}{\mathrm{seg}} ; K_{p}=-34,149 ; K_{i}=-5,6087
$$


Para el diseño del controlador se emplea el siguiente esquema de retroalimentación. En la Figura 2 se observa el diagrama de bloques de la implementación del controlador PID.

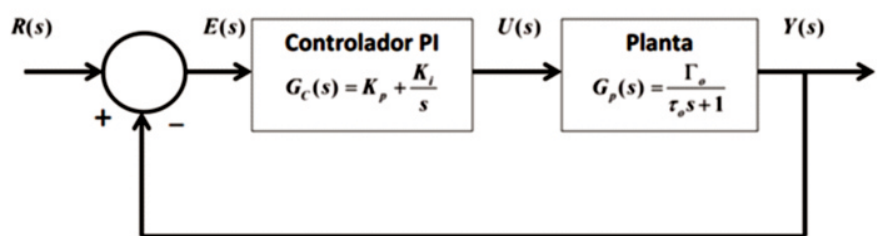

Figura 2. Diagrama de bloques control PID Fuente: elaboración propia.

La función de transferencia del sistema en lazo cerrado está dada por la ecuación (7):

$$
G_{(t)}=\frac{\left(K_{p} r_{0} / t_{0}\right) s+\left(K_{i} r_{0} / t_{0}\right)}{s^{2}+\left(L-\left(L-\left(K_{p} r_{0}\right)\right.\right.} \frac{s-\left(K_{i} r_{0}\right)}{t_{0}}
$$

Para el diseño del controlador, comparamos el polinomio en lazo cerrado con el polinomio característico deseado

$$
s^{2}+2 \rho \omega_{o} s+\omega_{o}^{2}
$$

De esta comparación obtenemos los parámetros del PI.

Para el diseño se seleccionó un tiempo de respuesta $t_{s}=30 \mathrm{seg}$, y un $p=0.7$. Los parámetros obtenidos fueron

$$
\omega_{0}=0,22 \frac{\mathrm{rad}}{\mathrm{sg}} ; K_{p}=-34,149 ; K_{i}=-5,6087
$$

Para la discretización del PID se aplicó la aproximación de Tustin.

$$
s=\frac{2}{T_{\mathrm{m}}} \frac{z-1}{z+1}
$$

La discretización del PID genera los parámetros listados en las ecuaciones (8) y (9) siguientes:

$$
G_{c}\left(z, T_{m}\right)=\frac{U(s)}{Y(s)} \mid s=\frac{2 z-l}{T_{m}{ }^{2}+l}=\frac{\beta_{0} Z^{2}+\beta_{l} Z+\beta_{2}}{Z^{2}+a_{l} z+a_{0}}
$$

Con:

$$
\begin{aligned}
& \beta_{0}=K_{p}+\frac{K_{l}+T_{m}}{2}+\frac{2 K_{D}}{T_{m}} \\
& \beta_{1}=K_{l} T_{m}+\frac{4 K_{D}}{T m} \\
& \beta_{2}=-K p+\frac{K_{l} T_{m}}{2}+\frac{2 K_{D}}{T_{m}} \\
& \propto_{1}=0 \\
& \propto_{2}=-1
\end{aligned}
$$$$
U\left(z, T_{m}\right)+\propto_{1} Z-1 U\left(z, T_{m}\right)+\propto_{2} Z^{-2} U\left(z, T_{m}\right)=
$$$$
B_{0} E\left(z, T_{m}\right)+B_{1} Z^{-1} E\left(z, T_{m}\right)+B_{2} Z^{-2} E\left(z, T_{m}\right)
$$

En la ecuación (10) se da la ecuación del controlador en términos de la transformada Z. En el dominio del tiempo, la ecuación (11) expresa de manera recursiva la implementación digital del PID

$$
\begin{aligned}
& U\left(k T_{m}\right)=-\propto_{1}, U\left(\overline{K-1} T_{m}\right)-\propto_{2} U\left(\overline{K-2} T_{m}\right) \\
& +\beta_{0} e\left(K T_{m}+\beta_{1} e\left(\overline{K-1} T_{m}+\beta_{2} e\left(\overline{K-2} T_{m}\right)\right.\right.
\end{aligned}
$$

En la Figura 4 se observan las gráficas de la concentración de gas bajo la acción del controlador PID digital.
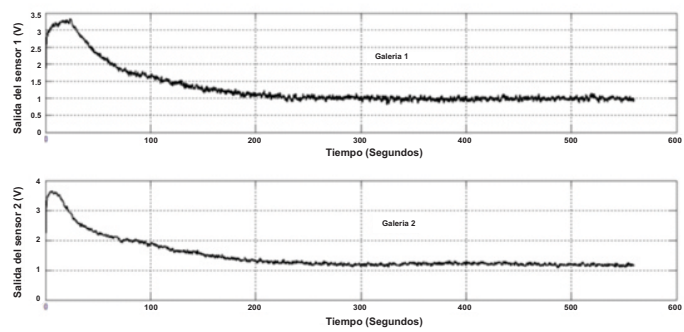

Figura 4. Señal de control ventilador auxiliar 1 Fuente: elaboración propia.

En las figuras 4 y 5 se observa el voltaje aplicado a los ventiladores y finalmente en la Figura 6 se observa la potencia consumida por los mismos.

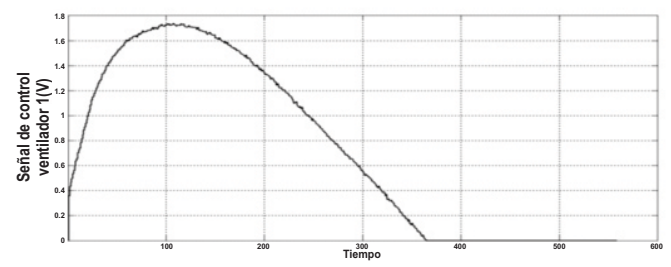

Figura 4. Señal de control ventilador auxiliar 1 Fuente: elaboración propia.

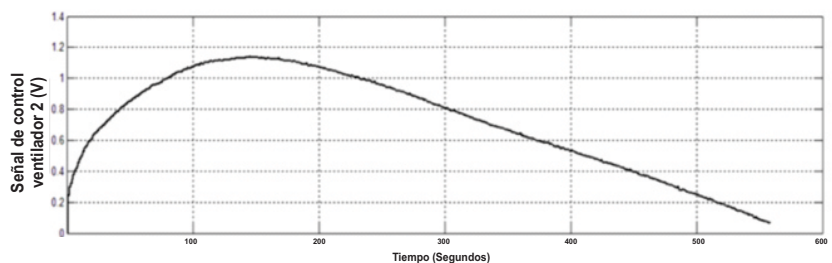

Figura 5. Señal de control ventilador 2 Fuente: elaboración propia.
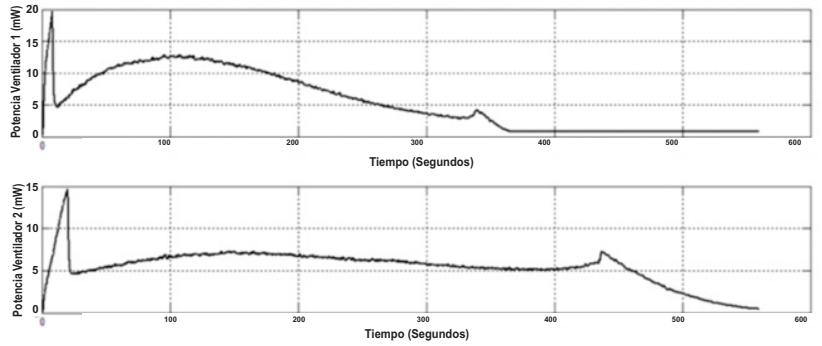

Figura 6. Potencia consumida por los ventiladores. Fuente: Elaboración propia

\section{CONTROL ON-OFF}

Para diseñar el controlador on/off, la entrada de control se genera aplicando los valores de voltaje máximos y mínimos disponibles a los ventiladores en respuesta a la diferencia entre las salidas de los sensores y los niveles deseados de gas con el objetivo de evacuar lo más rápido posible el gas y llevar los niveles a valores seguros. La ley de control para este caso está dada por:

$$
U\left(K T_{m}\right)=-G_{0} \operatorname{Sign}\left[y\left(K T_{m}\right)-y\left(K T_{m}\right)\right]
$$

Donde $\mathrm{y}\left(k T_{m}\right)$ es la salida deseada, representa el nivel de seguridad del gas en los frentes de extracción. 
$Y(k T m)$ es el nivel actual de gas en las galerías que proporcionan los sensores de gas.

$U(k T m)$ es la señal de control generada por la ley de control on/off.

En la Figura 7 se observan las salidas de los sensores de gas y la acción de los controladores frente a un repentino aumento de gas
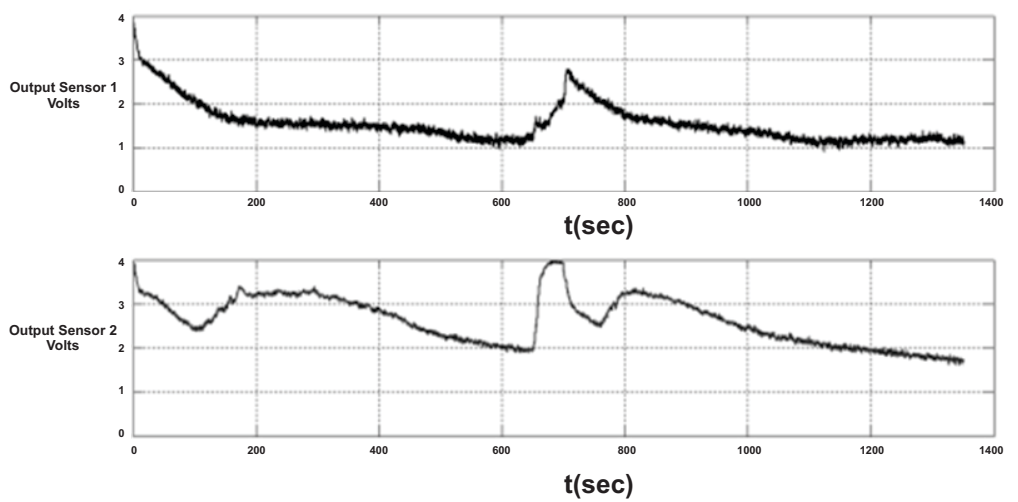

Figura 7. Salidas de los sensores de gas bajo control On-Off Fuente: elaboración propia.

\section{CONTROLADOR POR MODOS DESLIZANTES}

Para diseñar un controlador por modos deslizantes se debe proponer la siguiente superficie deslizante (ecuación (13))

$$
Z(t)=e(t)+\lambda ? e(t) d t
$$

Donde:

$e(t)=y(t)-y^{*}(t)$ es el error de seguimiento entre la salida actual $y(t)$ de los sensores de gas y la concentración deseada de gas (que puede estar por debajo del umbral mínimo permisible para mayor seguridad) $y^{*}(t) \lambda$ es una ganancia integral que asegura una convergencia a la superficie $z(t)$ de forma rápida.

La ley de control está dada por la ecuación 14 .

$$
U(t)=-G) \text { sign }[Z(t)] \quad(14)
$$

$\Gamma o=2.5$ es una ganancia de la ley de control y depende de los tamaños máximo y mínimo disponibles para la señal de control. Las salidas generadas por la aplicación de esta ley de control se muestran en la Figura 8, y la señal de control aplicada a los ventiladores se ilustra en la Figura 9 (ventilador 1) y en la Figura 10 (ventilador 2).
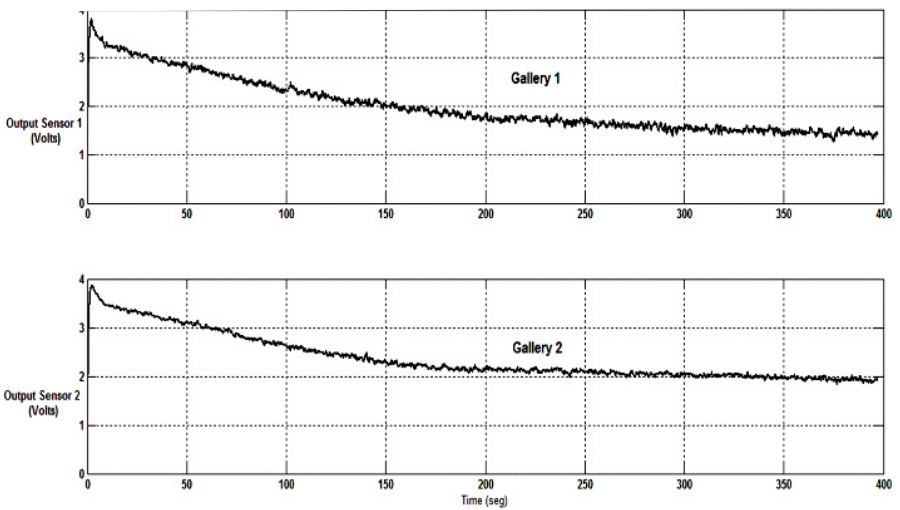

Figura 8. Salidas de los sensores de gas. Fuente: elaboración propia

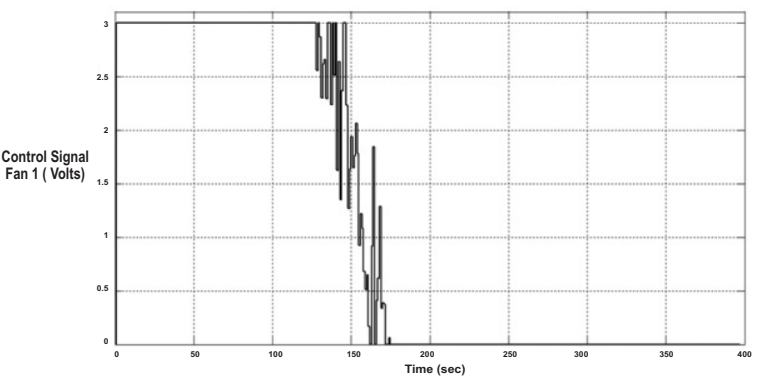

Figura 9. Señal de control aplicada al ventilador 1 Fuente: elaboración propia

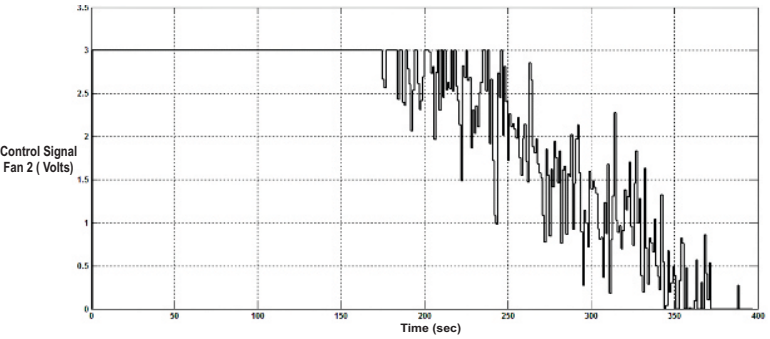

Figura 10. Señal de control aplicada al ventilador 2 Fuente: elaboración propia

\section{CONCLUSIONES}

Para el entendimiento de los fenómenos de la ventilación en minería subterránea es importante contar con prototipos reales que permitan simular condiciones de flujos, temperaturas y humedad en los ambientes confinados y que admitan aplicar diferentes estrategias de control para evaluar su funcionamiento.

El control PID es la estrategia más efectiva en la planta prototipo debido a su versatilidad y fácil implementación. En la vida real, dispositivos de control tipo PLC, HMI, variadores de velocidad permiten la implementación de controladores PID.

El control on-off es una de las estrategias más utilizadas por su facilidad de implementación, pero no presenta desempeños óptimos.

De la comparación de las estrategias de control aplicadas se evalúa que el control por modos deslizantes evacúa el gas de forma más rápida, pero exige una señal de control más fuerte, que cambia más precipitadamente con el tiempo.

El control PID proporciona una respuesta más lenta en la evacuación del gas y tiene una señal de control más suave.

Las pruebas de control permiten demostrar que la aplicación de estrategias de control al problema de la ventilación de minas, es una alternativa importante, ya que gracias al control se pueden extraer las concentraciones de gas generadas en los frentes de explotación en tiempos cortos, permitiendo tener un ahorro de energía sobre los consumos de energía de los ventiladores, ya que estos equipos solo estarán operando con su potencia máxima en los momentos de mayor nivel de gas. 


\section{REFERENCIAS}

Di Benedetto, M., D'Innocenzo, A., Serra, E. \& Witrant, E. (2008). Automatic verification of wireless control in a mining ventilation system. IEEE CASE Inveted paper.

Edwards, C. \& Spurgeon, S. K. (1998). Sliding Mode Control Theory and Applications. London: Pabstow UK.

Keith, G. \& Wallace, J.R. (s.f.). General operation characteristics and industry practices of mine ventilation systems. Fresno, California, United States of America: Mine Ventilation Services, Inc.

Olaru, S., Sandou, G., Witrant, E. \& Niculescu, S. (2008). Receding Horizon Climate Control in Metal Mine Extraction Rooms. IEEE CASE Invited Paper.

Reed, W. \& Taylor, C. (2007). Factors affecting the development of mine face ventilation systems in the 20th century. Pittsburgh: National Institute for Occupational Safety and Health.

Salamanca, J. M., Niño, J. \& Higuera, O. (2012). Sistemas de control automatico en la ventilación de minas. Importancia en la mineria subterranea y su desarrollo en Colombia. En IX Congreso Internacional de Electronica y Tecnologías de Avanzada. Cucuta, Colombia.

Salamanca, J. M., Niño, J. J. \& Pérez, A. F. (2014). Estrategias de control aplicadas a una mina prototipo para el estudio del problema de la ventilación de minas. Bogotá: Tecnura.
Viorel, B. (2011). Stabilization of Navier Stokes Flows. London: Springer Verlag.

Witrant, E. \& Johansson, K. H. (2008). Air flow modeling in deepwells:application to mining ventilation. 4th Congf.

Witrant, E., D' Innocenzo, A., Isaksson, A. J., Di Benedetto, M. D., Johansson, K. H., Santucci, F. \& Strand, M. (2008). Mining ventilation Control: a new industrial case for wireless automation. Washington DC, USA: IEEE Conference on Automation Science and Engineering.

Witrant, E., Niculescu, S. I. \& Marchand, N. (2008). A real-time control approach for mine climate regulation. 7th International Conference on Mathematical Problems in Engineering, Aerospace and Sciences (ICNPAA). Genova, Italy.

Yunan, H., Koroleva, O. I. \& Miroslav, K. (2003). Nonlinear control of mine ventilation networks. System \& Control Letter.

Yunan, H., Koroleva, O. \& Miroslav, K. (2003). Nonlinear control of mine ventilation networks. Systems \& Control Letters.

Zhu, W. \& Wang, L. (2013, June). Intelligent control of ventilation system for energy-efficient buildings with CO2 Predictive Model. Smart Grid, IEEE Transactions on. 